\title{
Hepatitis C: A Critical Analysis of Therapeutic Response Predictors
}

Focaccia R., Siciliano R.F., Santos E.D.B., Boccardo E., Conceição O.J.G., Barbosa U.O. and Galante V.C.
Emílio Ribas Infectious Diseases Institute, São Paulo, SP, Brazil

Treatment of chronic hepatitis $C$ is still unspecific. However, there is great expectancy concerning the new pegylated interferons. As there has been much controversy about the best parameters to determine whether treatment is effective, we analyzed several criteria currently used for evaluation, including serum alanine aminotransferase (ALT) normalization, viral load reduction and improvement of hepatic histology.

Key Words: ALT normalization, hepatitis C, viral load reduction.

Viral Hepatitis C (VHC) is currently one of the most important worldwide public health problems. Its global prevalence is estimated to be around 3\%, ranging from $0.1 \%$ to $5 \%$ [1]. Focaccia, et al. [2] found $1.42 \%$ seroprevalence for Hepatitis C Virus (HCV) in the city of São Paulo (2.2\% among the $31-40$ year-old group and $3.2 \%$ among the over 60 year-old group).

The incidence of new symptomatic cases in U.S. is $1-3$ cases/100,000/year. Considering that VHC is asymptomatic in most cases, it might become the most common pandemic disease of the millennium. Despite a reduction in post-transfusion infection due to an increasingly effective selection of blood donors, the number of individuals being diagnosed as carriers tends to rise, owing to: a) an increase in the consumption of injectable drugs worldwide; b) a lack of known risk factors for infection in half of the cases, making prevention recommendations difficult; c) millions of clinically silent cases worldwide infected during the last three decades who will be identified during the next 15 years.

Received on 4 July 2001; revised 25 March 2002.

Address for correspondence: Dr. Roberto Focaccia. Av. Higienópolis 360, ap14, São Paulo, SP, Zip Code: 01238-001, Brazil.E-mail:focaccia@uol.com.br

The Brazilian Journal of Infectious Diseases 2002;6(2):74-81 (C) 2002 by The Brazilian Journal of Infectious Diseases and Contexto Publishing. All rights reserved. 1413-8670
Around $85 \%$ of the acute $\mathrm{HCV}$ infections become chronic and liver cirrhosis develops in about $25 \%$ of cases, whereas $1 \%$ to $4 \%$ of those may develop hepatocarcinoma. Consequently, the massive effort being conducted for the development of new and efficient therapeutic approaches is completely justifiable.

Hepatitis C Virus is an RNA virus which may have certain degrees of transcription modifications in its genome, generating variants, defined as quasi-species. These are frequently developed under immune system pressure, as an effort to escape from hostile conditions and to perpetuate. In fact, HCV comprises a large complex of quasi-species, probably responsible for the high incidence of chronic infections and for drug resistance $[3,4]$. It is known that the high incidence of viral resistance - both to the immune system and to drugs - is due to the ability of HCV to generate variants and to replicate outside the liver [5-7].

On the other hand, infected individuals can have fibrinogenic viral mutants (angiotensin and growth transformation factors - TGF-beta- 1 - producers) which oppose interferon's mechanism of action [8].

In theory, $\mathrm{HCV}$ could be eradicated from the human organism, since it is, as has been said, an RNA virus. However, despite the great promise of pegylated interferon, therapy is still unspecific, lengthy, difficult to apply, and very frequently has precarious and temporary results. There are reports that treatment of $\mathrm{HCV}$ improves survival [9] "but 
such reports do not stem from long term follow-up of randomized controlled trials-rather a comparison of outcome in subjects treated or not treated, thus selection bias cannot be avoided"(Heathcote J [10]).

Massive effort made worldwide in a search for adequate therapeutic schemes has generated many controversies, namely: a) the actual efficacy of the drugs; b) doubtful predictive parameters of favorable therapeutic response; c) questionable criteria for evaluation of drug efficacy; d) adequate dosage schedules; e) types of drug associations; f) treatment timing, including exact moments of therapeutic start and interruption; g) exact inclusion/exclusion treatment criteria; h) cost-benefit of therapy; i) treatment of the extra-hepatic complications; $j$ ) indications for re-treatment; $\mathrm{k}$ ) adequate laboratory monitoring, among others.

The aim of this analysis is to evaluate controversies and divergences, as well as critically discuss criteria used for VHC treatment indication, monitoring and efficacy.

\section{Criteria for Evaluation of Results}

There have been many controversies over the best parameters for the prediction and interpretation of results. Factors associated with an unfavorable therapeutic response include: 1) viral genotype; 2) cirrhosis; 3) HCV-HIV co-infection; 4) elevated iron overload; 5) high viral load; 6) immunosuppressive conditions, such as alcoholism, diabetes, organ transplants and illicit drug abuse; 7) resistant HCV mutations; 8) gender (male); 9) age (above 50 years old); 10) morbid obesity; and 11) probable individual immune-genetic factors.

However, an essential question that has been raised is that not every factor significantly associated with favorable results can be considered predictive. Therefore, denial of access to treatment based solely on the presence of one or more factors significantly associated with inefficient therapeutic response does not seem ethical to us.
Most clinical drug trials employed different definitions of therapeutic efficacy, obviously giving noncomparable results. Hepatic function tests and serum alanine aminotransferase (ALT) normalization, viral load reduction and hepatic histology analysis improvement are usually used to evaluate therapeutic efficacy. It is doubtful if responses measured by various parameters can equally predict the development of complications, such as cirrhosis, hepatic insufficiency, hepatocarcinoma or death related to $\mathrm{VHC}[11]$.

ALT normalization. The definition of sustained response is a major controversy in chronic VHC treatment, even though it is essential to assess therapeutic efficacy. The definition made by the National Institute of Health Consensus, USA, of the efficacy of therapy in HCV treatment includes ALT normalization and/or RNA reduction [12]. It has the most widely used by investigators, however this definition is limited due to important new concepts about HCV. Initially, sustained response was defined as normal ALT levels for more than six months after cessation of therapy. This period has been extended to twelve months in more recent studies and currently some protocols even consider a 24 month follow-up period.

However, ALT levels frequently oscillate in chronic hepatitis; hence, they do not accurately reflect the type and the extension of histological lesions. In addition, this enzyme may rise due to several other extra-hepatic clinical conditions. Only repeatedly dosed ALT (transaminase curve) should be considered [13]. Yet, about $25 \%$ of HCV-positive patients have persistently normal ALT.

Meta-analysis evaluation of several studies indicate that about $85 \%$ of patients with normal ALT levels have mild necro-inflammatory activity, while there is moderate activity in $10 \%$ and normal histopathological signs in about $5 \%$. Also, although fibrosis is commonly absent, cirrhosis or advanced stage lesions are occasionally found [14-23].

Cirrhotic patients treated with a combination of interferon and ribavirin may clear the hepatitis $\mathrm{C}$ virus without normalization of ALT levels [24]. So, in patients with cirrhosis, ALT levels may be a less appropriate endpoint in the measurement of response to therapy. 
Temporary pre or post-treatment alterations in ALT may indicate only an inflammatory and/or intracellular cholestatic component [25-28]. Pre-treatment ALT level reduction may be associated with drug action (immune modulator and/or antiviral and/or antiinflammatory and/or anti-fibrosis), but it cannot be used as the sole efficacy parameter. It has been known for over two decades that ribavirin in monotherapy often promotes a reduction or normalization of ALT solely during the period that it is administered. Hence, the isolated use of ALT as an indication of therapeutic success is extremely questionable.

HCV-RNA negativation. Serum HCV RNA levels correlate with hepatic HCV RNA [29]. Viremia may attain levels ranging from less than $200 \mathrm{IU}$ RNA/ml frequently undetectable by current molecular methods - to very high levels. They frequently vary between $10^{3}$ and $2 \times 10^{6}$. Spontaneous oscillations may occur during several decades after primary infection. Values higher than $800,000 \mathrm{UI} / \mathrm{RNA} / \mathrm{ml}$ have been considered significantly high, being associated with unfavorable responses to therapy. There is no correlation between viral load and the histological lesion stage. Quite frequently there are high viral loads and minimal histological damage or vice-versa [30,31].

Viral load may be influenced by multiple factors such as: age at infection; gender; duration of illness; viral type/subtype; mode of viral acquisition; and the quantification method employed [32].

Therefore, viral load levels have to be examined cautiously due to the possibility of oscillations unrelated to treatment. During the pre-treatment period, sudden, unexpected, repeated and conflicting variations may occur due to technical errors, environmental molecular lab contamination, or individual variations in immune status. Viral loads obtained through different techniques do not give comparable results.

A viremia rebound, with significant transient elevations, may occur after the cessation of medication. Such a phenomenon is caused by the extended inhibition of the immune system through medication pressure, which does not necessarily imply in a therapeutic failure.
Hence, the clinical value of viral load measurements as a predictive factor and as an efficacy parameter of therapy is also a controversial matter. Multivariate analysis was made of 8 studies selected out of 18, involving 988 patients. This analysis included studies that used ALT normalization and viral load negativation as their definition of complete and sustained response. It was concluded that viral load negativation achieved after 4 weeks of interferon monotherapy had a high predictive value. It was suggested that treatment cessation should occur in case no RNA/HCV load reduction was achieved [33]. The above-mentioned study did not consider the hepatic biopsy relevant. Although the authors suggest that treatment should be maintained for 12 months, regardless of other parameters, whenever the fibrogenesis score in a pre-treatment biopsy is greater than or equal to $\mathrm{F}_{2}$ by Metavir graduation (or the correspondent category in other classifications).

Some authors suggest that early, abrupt reductions of viral load may have a predictive value for sustained response [34,35] and might indicate a reduction in the risk of the development of hepatocarcinoma. However, regression of hepatic fibrosis may occur due to the use of interferon, even in the absence of viral load negativation or reduction [36]. On the other hand, patients who had reduced viremia did not have reduced histopathological lesions. Occasionally some degree of progression was detected.

Therefore, the clinical value of viral load quantification as a predictive factor and as a parameter of therapeutic efficacy is also a controversial matter. Viral load negativation or reduction during treatment may indicate drug activity, but it does not necessarily insure real efficacy. From such observations, it appears that the premature cessation of therapy when there is no viral load negativation or reduction is not scientifically grounded.

Improvement in hepatic histology. Hepatic fibrosis stages are representative of disease progression. Mortality and complications are related to cirrhosis. Poynard et al. [30] found a strong, almost linear, correlation of fibrosis stages with age at biopsy and 
duration of infection. So, although HCV is a viral disease, it is mainly a fibrotic disease.

The great majority of medical studies [37-50] use ALT level normalization and undetectable viral load as a way to evaluate efficacy of a given therapeutic scheme. Few clinical studies have conducted histopathological exams on hepatic tissues, using them as a measure of therapeutic efficacy. Even studies that follow this procedure still use ALT level and viral load as indicators of therapeutic efficacy [44,45,51]. These studies included a limited number of patients and were based solely on interferon monotherapy.

Meta-analysis of 17 published clinical studies that included 1,223 patients indicated that when ALT was altered, there was no histological improvement with regression of hepatic fibrosis measured by the Knodell classification [52]. Despite the historical value of the Knodell classification, this criterion is not very sensitive, nor is it linear, and therefore it might not exclude lesions already considered cirrhotic [53]. Furthermore, other authors [30, 54-56] found no correlation between the concomitant improvement of biochemical, viral and histological parameters.

According to Poynard and coworkers [53], when they administrated interferon monotherapy for 18 months, patients with a sustained therapeutic response for 1 to 12 years had a regression in fibrosis of $189 \%$ per year. Septal fibrosis had disappeared in $56 \%$. There was also lesion reduction in $16 \%$ of the patients who had cirrhosis. Of those with no sustained response, $66 \%$ had regression of septal fibrosis and only $15 \%$ showed progression. These results have led to the conclusion that interferon improves fibrosis progression dramatically, even without sustained response and independent of genotypic, biochemical or premature viral response.

Several recent randomized clinical studies have also documented the fact that sustained histological response is common among patients without viral clearance [30,57-60]. Furthermore, it was found that the maintenance of treatment in premature non-responsive patients can reduce hepatic fibrosis in the long run [6167], and perhaps the risk of development of hepatocarcinoma related to $\mathrm{HCV}[58,68-71]$. In the same way, pegylated interferon seems to improve the histological response when compared with standard therapy [72,73].

Interferon apparently affects hepatic fibrogenesis mechanisms [74,75], a hypothesis supported by serum alterations of the type III pro-collagen $\mathrm{N}$ terminal pro-peptide, considered a fibrogenesis marker in HCV patients' [76-79], activation of human type I collagen gene expression by transforming growth factor-beta-1 [31,80], alterations in the matrix of subendothelial space and oxidative stress [81]. Although the antiproliferative mechanisms of interferon remain poorly understood there is clear evidence of growth inhibition of hepatocellular carcinoma. Murphy et al. showed the effect of interferon on the inhibition of liver carcinoma, specifically by S-phase progression, most likely because of the inhibition of cyclin A induction, resulting in decreased activity of the associated $\mathrm{Cdk} 2$ and $\mathrm{Cdc} 2$ kinase [82].

Poynard et al. [83] related three factors independently associated with significant fibrosis reduction: sustained viral response, duration of treatment, and baseline fibrosis stage after therapy with combination of interferon and ribavirin. This study also concludes that independent of virological response, treatment duration and baseline viral load is associated with fibrosis improvement.

Another observation that should be considered is the sustained histological improvement in patients treated with alpha-1-thymosin (a strong immune modulator) for several years after the cessation of therapy [84], though its effects on morbidity and mortality remain to be evaluated.

The variability in the results reinforces the controversy over the definition of a complete sustained response, the endpoint of clinical drug trials and the predictive value of the parameters used (ALT normalization and viral load negativation).

The hepatic histopathological evaluation made after the end of interferon and ribavirin therapy seems to be of great importance, which had not been ascribed in initial studies. This relies ultimately on the real therapeutic benefit, which involves histopathological hepatic improvement. 
In a somewhat rigorous analysis, most published studies are bound to be criticized due to: a) patients with different degrees or stages of histopathological lesions; b) no similarity among inclusion and exclusion criteria for patients in the study; c) no comparison between pre- and late post-treatment biopsies; d) frequent use of monotherapy; e) rather small samples; f) dissimilar patterns among study designs, such as diverse clinical end points and sustained response definitions.

Ferenci [85] reviewed and identified 115 randomized clinical trials and 7 meta-analyses of interferon therapy in chronic VHC between 1989 and 1999 , all of them full published papers. Only 20 trials prospectively studied and compardc different interferon doses. In line with these data, it appears that few studies can be considered conclusive.

Despite the great number of studies, we do not know all the mechanisms of action and can not develop ideal therapeutic schemes. It would be necessary to carry out prospective, easily reproduced and long-term follow-up studies, as well as to evaluate all interfering variables and to compare various schemes with different dose regimens.

The fibrosis stage summarizes the vulnerability of a patient and is predictive of progression to cirrhosis [30]. Therefore, fibrosis is the gold standard parameter to indicate treatment efficacy. For good comparisons between fibrosis rates before and after the end of treatment, to plan subsequent management strategies, it is necessary to perform a later second liver biopsy, 18 to 24 months later.

Pre- and post-treatment liver biopsy seems to have a real value in the evaluation of therapeutic results, which leads us to believe that reduction in histopathological lesions should be included in the definition of a complete sustained response.

Shiratori and coworkers [86], in a retrospective cohort study comparing multi-center results in Japan involving hundreds of patients treated with interferon for many years, concluded that there was a high percentage of hepatic fibrosis regression in patients who had sustained viral response. Furthermore, this study found that treated patients, without a sustained viral response, also had a reduction of fibrosis lesions when checkedseveral years later, even though at a slightly lower frequency.

The studies of Shiratori et al. [86] and Schiffman et al. [57] prompted the initiation of the "Hepatitis C Antiviral Long-Term Treatment Against Cirrhosis (HALT-C)" National Institute of Health-supported trial to evaluate long-term pegylated interferon in nonresponders to previous antiviral therapy. The results of this study will not be available for many years.

Everson et al. [87] showed that liver cirrhosis HCV patients benefit from interferon therapy not only due to clearance of virus but because of improvement in liver histology. After therapy, fibrosis reduction continues for a long time.

Based on present knowledge our group has already adopted the criterium of liver biopsy evaluation, conducted before treatment and 18-24 months after the cessation of treatment. Drug therapy is also maintained for 12-18 months, even when there is no premature biochemical or viral response.

\section{References}

1. EASL International Consensus Conference on Hepatitis C. J Hepatol 1999;31 (supl.1): 3-264.

2. Focaccia R., et al. Estimated Prevalence of Viral Hepatitis in the General population of the Municipality of São Paulo, Measured by a Serologic Survey of a Stratified, Randomized and Residence-Based Population. Br J Infect Dis 1998;2:269-83.

3. Domingo E. Biological Significance of Viral Quasispecies. Viral Hepatitis Reviews 1996;2(4):247-61.

4. Farci P., et al. The Outcome of Acute Hepatitis C Predicted by the Evolution of the Viral Quasispecies. Science 2000;288:339-43.

5. Thomas H.C., et al. Possible mechanisms of action and reasons for failure of antiviral therapy in chronic hepatitis C. J Hepatol 1999;31(supl. 1): 52-9.

6. Focaccia R. Tratado de Infectologia, Rio de Janeiro: Atheneu 1997:1-182.

7. De Francesco R. Molecular virology of the hepatitis C virus. J Hepatol 1999;31(supl. 1);47-53.

8. Powell E.E., et al. Host Genetic Factors Influence Disease Progression in Chronic Hepatitis C. Hepatology 2000;31(4):828-33. 
9. Niedeau C., et al. Prognosis of chronic hepatitis C: Results of a large, prospective cohort study. Hepatology 1998; $28: 1687-95$.

10. Heathcote J. Sem Liver Disease 2000;20(2):185-99.

11. Editorial. Interferon-alpha for chronic hepatitis $\mathrm{C}$ : reducing the uncertainties. Ann Intern Med 1997;127(10):918-20.

12. NIH Consensus Statement. Management of hepatitis C. Hepatology 1997; 15:1-41.

13. Di Bisceglie A.M. Chronic hepatitis $C$ viral infection in patients with normal serum alanine aminotransferases. Am J Med 1999;107(6B):53S-5S.

14. Shindo M., et al. The virological and histological states of anti-hepatitis $\mathrm{C}$ virus positive subjects with normal liver biochemical values. Hepatology 1995;22:418-25.

15. Marcellin P., et al. Therapy of hepatitis C: Patients with normal aminotransferase levels. Hepatology 1997;26(supl.1):133S-6S.

16. Rossini A., et al. Virological response to interferon treatment in hepatitis $\mathrm{C}$ virus carriers with normal aminotransferase levels and chronic hepatitis. Hepatology 1997;26:1012-7.

17. Sangiovanni A., et al. Interferon alpha treatment of HCV RNA carriers with persistently normal transaminase levels: a pilot randomized controlled study. Hepatology 1998;27:853-6.

18. Longo F., et al. Virological and histological effects of interferon treatment in $\mathrm{HCV}$ positive patients persistently normal ALT levels. Hepatology 1998; 28:705A.

19. Shakil A.O., et $a$ l. Volunteer blood-donors with antibody to hepatitis $\mathrm{C}$ virus: clinical, biochemical, virologic, and histologic features. The Hepatitis C Study Group. Ann Intern Med 1995; 123:330-7.

20. Matthurin P., et al. Slow progression rate of fibrosis in hepatitis $\mathrm{C}$ virus patients with persistently normal alanine transaminase activity. Hepatology 1998;27:868-72.

21. Pouti C., et al. Clinical, histological, and virology features of hepatitis $\mathrm{C}$ virus carriers either persistently normal or abnormal alanine transaminase levels. Hepatology 1997;26:1393-8.

22. Naito M., et al. Hepatitis $C$ viral quasispecies in hepatitis $\mathrm{C}$ virus carriers with normal liver enzymes and patients with type chronic liver disease. Hepatology 1995;22:407-12.

23. Gholson C.F., et al. Chronic hepatitis $\mathrm{C}$ with normal aminotransferase levels. A clinical histologic study. Am J Gastroenterol 1997;92:1788-92.

24. Puoti C., Magrini A., Stati T., et al. Clinical, histological, and virological features of hepatitis $\mathrm{C}$ virus carriers with persistently normal or abnormal alanine transaminase levels. Hepatology 1997;26:1393-8.
25. Poynard T., et al. Hepatitis C: Somber views of natural history and optimistic views of interferon treatment. Hepatology 1998;27:1443-4.

26. Nishigushi S., et al. Randomized trial of effects of interferon alpha on incidence of hepatocellular carcinoma in chronic active hepatitis $\mathrm{C}$ with cirrhosis. Lancet 1995;346:1051-5.

27. Camps J., et al. Prediction of sustained remission of chronic hepatitis $\mathrm{C}$ after a 12 months course of alpha interferon. J Hepatol 1994;21:4-11.

28. Marcellin P., et al. Recombinant human alpha interferon in patients with chronic non-A, non-B hepatitis: a multicenter randomized controlled trial from France. Hepatology 1991;13:393-7.

29. Deguzman L.J., et al. Plasma hepatitis C RNA is comparable to hepatic HCV RNA when obtained while on therapy at the end of treatment as a predictor of sustained response in patients with chronic hepatitis $\mathrm{C}$ infection. Gastroenterology 2001;120:A-30.

30. Poynard T., et al. Fibrosis in Patients with Hepatitis $\mathrm{C}$ :Detection and Significance. Sem Liver Disease 2000;20(1):47-55.

31. Roulot D., et al. Quantitative Analysis of Transforming growth factor Beta 1 Messenger RNA in the liver of patients with chronic hepatitis $\mathrm{C}$ : Absence of correlation between high levels and severity of diseases. Hepatology 1995;21(2):298-304.

32. Fanning L.J., et al. The hepatitis $\mathrm{C}$ virus: Master of diversity and challenging adversary. Internet: medscape Gastroenterology, 2000. Site: http:// id.medscape.com/Medscape/gastro/jo...02/ mgi6558.fann/pnt-mgi6558.fann.html.

33. Civeira M.P., et al. Early predictors of response to treatment in patients with chronic hepatitis C. J Hepatol 1999;31(supl.1):237-43.

34. Rossini A. Early hepatitis C virus (HCV) RNA kinetic during interferon (IFN)therapy: relationship to treatment schedule and outcome. Antiviral Therapy 2000;5(supl. 1):C100.

35. Puoti M. Interferon $5 \mathrm{UM}$ daily in combination with ribavirin is more effective than TW schedules in the treatment of patients with hepatitis $\mathrm{C}$ non-responders to interferon monotherapy. Antiviral Therapy 2000;5(supl. 1):C100.

36. Shiratori Y., et al. Histologic improvement of fibrosis in patients with hepatitis $\mathrm{C}$ who have sustained response to interferon therapy. Ann Intern Med 2000;132(7):517-24.

37. Craxi A. Definition of Response to antiviral therapy in chronic hepatitis C. J Hepatol 1999;31(supl.1):160-7.

38. Chayama K., et al. Antiviral effect of lymphoblastoid interferon alpha on hepatitis $\mathrm{C}$ virus in patients with chronic hepatitis type C. J Gastroenterol Hepat 1994;9:128-33. 
39. Vento S., et al. Lack of sustained efficacy of interferon in patients with chronic hepatitis C. N Engl J Med 1966;334:1479-80.

40. Barnes E., et al. Long-term efficacy of treatment of chronic hepatitis $\mathrm{C}$ with alpha interferon or alpha interferon and ribavirin. J Hepatol 1999;31(supl. 1):244-9.

41. Manesis E.K., et al. Biochemical and virological outcome of patients with chronic hepatitis $\mathrm{C}$ treated with interferon alpha-2b for 6 or 12 months: A 4 year followup of 211 patients. Hepatology 1997;26:734-7.

42. Biden K., et al. Frequency and mutation and deletion of the tumor suppressor gene CDKN2A (MTS1/p16) in hepatocelular carcinoma from an Australian population. Hepatology 1997;25:593-7.

43. Saracco G., et al. Long-term follow-up of patients with hepatitis $\mathrm{C}$ treated with different doses of interferon alpha-2b. Hepatology 1993;18:1300-5.

44. Vento S., et al. Lack of sustained efficacy of interferon in patients with chronic hepatitis C. N Engl J Med 1996;334:1479-80.

45. Marcellin P., et al. Long term histologic improvement and loss of detectable intrahepatic HCV RNA in patients with chronic hepatitis $\mathrm{C}$ and sustained response to interferon alpha therapy. Ann Intern Med 1997; 127:875-81.

46. Castillo I., et al. Virological and biochemical long-term follow-up of patients with chronic hepatitis $\mathrm{C}$ treated with interferon. Hepatology 1994; 19:1342-6.

47. Lau D.T., et al. 10-year follow after interferon-alpha therapy for chronic hepatitis C. Hepatology 1998;28:1121-7.

48. Cammà C., et al. Long-term course of interferon-treated chronic hepatitis C. J Hepatol 1998;28:531-7.

49. Chemello L., et al. Persistent hepatitis $\mathrm{C}$ viremia predicts late relapse after sustained response to interferonalpha in chronic hepatitis C. Ann Intern Med 1966; $124: 1058-60$.

50. Shiffman M.L., et al. Relationship between biochemical, virologic and histologic response during interferon treatment of chronic hepatitis C. Hepatology 1997;26:780-5.

51. Duchatelle V., et al. Changes in liver fibrosis at the end of alpha interferon therapy and 6 to 18 months later in patients with chronic hepatitis $\mathrm{C}$ : quantitative assessment by a morphometric method. J Hepatol 1998;29:20-8.

52. Cammà $\mathrm{C}$., et al. The effect of interferon on the liver in chronic hepatitis $\mathrm{C}$ : a quantitative evaluation of histology by meta-analysis. J Hepatol 1997;26:1187-99.

53. Poynard T., et al. Effect of interferon therapy in "non responder"patients with chronic hepatitis C. J Hepatol 1999;31(supl.1):178-83.
54. Hagiwara H., et al. Quantification of hepatitis C virus RNA in serum of assymptomatic blood donors and patients with type $\mathrm{C}$ chronic liver disease. Hepatology 1992; $17: 550$

55. Bruno S., et al. Post treatment natural history of subjects with chronic hepatitis $\mathrm{C}(\mathrm{CHC})$ showing sustained biochemical response (SR) to interferon-(IFN) therapy. Hepatology 1998;28:578-A.

56. Lau J.Y.N., et al. Significance of serum hepatitis $C$ virus RNA levels in chronic hepatitis C. Lancet 1993;341:1501-4.

57. Schiffman M.L., et al. A randomized control trial of maintenance interferon therapy for patients with chronic hepatitis $\mathrm{C}$ and persistent viremia. Gastroenterology 1999;11:1164-1172.

58. Yoshida H., et al. Interferon therapy reduces the risk for hepatocellular carcinoma: National surveillance program of cirrhotic and non cirrhotic patients with chronic hepatitis C in Japan. Ann Intern Med 1999;131:174-181.

59. Shindo M., et al. The clinical and virological characteristics and long-term outcome of HCV-RNA-positive long term responders following interferon therapy. Abstract. $50^{\text {th }}$ Annual Meeting, AASLD, Dallas, USA, 1999.

60. Shioda A., et al. Long-term prognosis of hepatocellular carcinoma developing after treatment of interferon in patients with chronic hepatitis C. Abstract. $50^{\text {th }}$ Annual Meeting, AASLD, Dallas, USA, 1999.

61. Cammà C., et al. Long-term course of interferon treated chronic hepatitis C. J Hepatol 1998;28:531-7.

62. Lindsey K.L., et al. Sustained histologic response in common in the absence of virologic response to interferon therapy for chronic hepatitis C. Gastroenterology 1998; 114:A-3.

63. Shiffman M.L., et al. The effect of maintenance interferon in chronic hepatitis $\mathrm{C}$ non responders: result of a randomized controlled trial. Gastroenterology 1998; 114:A-3.

64. Tanaka H., et al. Effect of interferon therapy on the incidence of hepatocellular carcinoma and mortality of patients with chronic hepatitis C. Antiviral Therapy 2000;5(supl. 1):C.92.

65. Sobesky R., et al. Modeling the impact of interferon alpha treatment on liver fibrosis progression in chronic hepatitis C. Gastroenterology 1999;116:378-86.

66. Yoshida H., et al. Interferon therapy reduces the risk for hepatocellular carcinoma: National Surveillance Program of Cirrhotic and Noncirrhotic Patients with Chronic Hepatitis C in Japan. Ann Intern Med 1999;131:174-81.

67. Sobesky R., et al. Modeling the Impact of Interferon Alpha treatment on Liver Fibrosis Progressions in Chronic hepatitis C: A Dynamic View. Gastroenterol 1999;116:378-86. 
68. Nishiguchi S., et al. Randomized trial of effect of interferon alpha on incidence of hepatocelullar carcinoma in chronic active hepatitis $\mathrm{C}$ with cirrhosis. Lancet 1995;346:1051-5.

69. Imai Y., et al. Relation of Interferon Therapy and Hepatocarcinoma in Patients with Chronic Hepatitis C. Ann Inter Med 1998; $129: 94-9$.

70. Mazzella G., et al. Alpha interferon treatment may prevent hepatocellular carcinoma in $\mathrm{HCV}$-related liver cirrhosis. J Hepatol 1996;24:141-7.

71. Schalm S.W., et al. Therapy of hepatitis C: patients with cirrhosis. Hepatology 1997;3(supl. 1):128S-32S.

72. Balart L.A., et al. Histologic improvement following treatment with once weekly pegylated interferon alpha-2A and thrice weekly interferon alpha $2 \mathrm{~A}$ in patients with chronic hepatitis $\mathrm{C}$ and compensated cirrhosis. Digest Diseases Week 2000, San Diego, USA, Abstract 978.

73. Heathcote E.J., et al. Pegylated interferon alpha-2a is superior to interferon alpha $2 \mathrm{a}$ in improvement posttreatment histologic outcome in chronic hepatitis $\mathrm{C}$ patients. Hepatology 2000;32:223A.

74. Mallat A., et al. Interferon alpha and gamma inhibit proliferation and synthesis of human cells Ito cells in culture. Hepatology 1995;21:1003-1010.

75. Poynard T., et al. Impact of interferon alpha- $2 b$ and ribavirin on progression of liver fibrosis in patients with chronic hepatitis C. Hepatology 2000;32:1131-1137.

76. Serejo F., et al. Interferon improves liver fibrosis in patients with chronic hepatitis $\mathrm{C}$ : The clinical significance of serum N-terminal propeptide of procollagen type III. 10 the International Symposium on Viral Hepatitis and Liver Disease, CDC, April 9-13, 2000, Atlanta, USA. Abstract Book: C92, Abstract C174.

77. Pilette C., et al. Effects anti-fibrosants des interférons. Gastroenterol Clin Biol 1997;21:466-71.

78. Czja C., et al. Differential effects of gamma interferon on collagen and fibronectin gene expression. J Biol Chem 1987;262:13348-51.

79. Goldring M., et al. Immune interferon suppresses levels of procollagen mRNA and type collagen synthesis in culture human articular and costal chondrocytes. J Biol Chem 1986;261:9049-56.

80. Reginato A., et al. Transcriptional modulation of cartilagespecific collagen gene expression by IFN and tumor necrosis factor alpha in culture human chrondocytes. Biochem J 1993:294:761-9.

81. Brown K.E. An Overview of Hepatic Fibrogenesis. Viral Hepatitis Reviews 2000;6(1-4):5-27.

82. Murphy D., et al. Interferon-alpha delays S-phase progression in human hepatocellular carcinoma cells via inhibition of specific cyclic-dependent kinase. Hepatology 2001;33(2):346-56.
83. Poynard T., et al. Impact of Interferon Alpha-2b and Ribavirin on Progression of Liver Fibrosis in Patients with Chronic Hepatitis C. Hepatology 2000;32(5):1131-7.

84. Sherman K.E. Combination therapy with thymosin alpha 1 and interferon for the treatment of chronic hepatitis $\mathrm{C}$ infection: a randomized, placebo-controlled doubleblind trial. Hepatology 1998;27:1128-35.

85. Ferenci P. Have we Achieved the Optimal Dosing of Interferon in Hepatitis C? Viral Hepatitis Reviews 1999;5(4):229-45.

86. Shiratori I. Histologic Improvement of Fibrosis in Patients with Hepatitis C Who Have Sustained Response to Interferon Therapy. Ann Intern Med 2000;132(7):517-24.

87. Everson G.T., et al. Efficacy of Interferon Treatment for Patients with Chronic Hepatitis C: Comparison of Response in Cirrhotics, Fibrotics, or Non-Fibrotics. Hepatology 1999;30(1):271-6. 